\title{
EVOLUÇÃO DA MORTALIDADE POR DOENÇAS DO APARELHO CIRCULATÓRIO EM UM MUNICÍPIO MINEIRO
}

\section{EVOLUTION OF MORTALITY BY DISEASES OF THE CIRCULATORY APPARATUS IN A MUNICIPAL MINEIRO}

\section{EVOLUCIÓN DE LA MORTALIDAD POR ENFERMEDADES DEL APARATO CIRCULATORIO EN UN MUNICIPIO MINEIRO}

\author{
Lucas Costa Cardoso Silva ${ }^{1}$, Naedson Rosa ${ }^{2}$, Álvaro da Silva Santos ${ }^{3}$, Luan Augusto Alves Garcia ${ }^{4}$
}

Fontes de financiamento: Coordenação de Aperfeiçoamento de Pessoal de Nível Superior (CAPES) - Bolsa de Doutorado.

\section{RESUMO}

Objetivo: analisar a mortalidade por doenças do aparelho circulatório em um município do interior mineiro. Método: Trata-se de estudo ecológico e série temporal por coeficiente de mortalidade e análise de tendência. Foram incluídos os registros de óbitos do capítulo IX Doenças do aparelho circulatório (CID-10: I00-I99) entre 1996 a 2014, disponíveis no Sistema de Informação de Mortalidade, sendo empreendia análise exploratória descritiva das variáveis, além de análise de tendência temporal. Resultados: os óbitos para o sexo masculino (Coeficiente de mortalidade médio: 82,2 x $10^{4}$ habitantes) e faixa etária $\geq 80$ anos (Coeficiente de mortalidade médio: 930,6 x 104 habitantes) foram mais expressivos. O coeficiente de mortalidade geral $(p=0,008)$ e por sexo (masculino e feminino $-p<0,001$ ) apresentaram evolução temporal crescente. Conclusão: Evidencia-se a importância do reconhecimento do padrão de mortalidade por doenças do aparelho circulatório como problema de saúde pública e a criação de ações de prevenção destes agravos com adoção de estilos de vida saudáveis.

Descritores: Mortalidade; Sistema Cardiovascular; Estudos Epidemiológicos; Estudos de Séries Temporais.

\begin{abstract}
Objective: to analyze the mortality due to diseases of the circulatory system in a municipality of the interior of Minas Gerais. Method: This is an ecological and time series study by mortality coefficient and trend analysis. Death registries of chapter IX - Circulatory diseases (ICD-10: I00-I99) between 1996 and 2014, available in the Mortality Information System. A descriptive exploratory analysis of the variables was undertaken, as well as a temporal trend analysis. Results: male deaths (mean mortality coefficient: $82.2 \times 10^{4}$ inhabitants) and age
\end{abstract}

\footnotetext{
${ }^{1}$ Bacharel em Educação Física pelo Centro de Ensino Superior de Uberaba (CESUBE)/Faculdade de Ciências Econômicas do Triângulo Mineiro (FCETM)

2 Bacharel em Administração. Faculdade de Ciências Econômicas do Triângulo Mineiro - FCETM e Centro de Ensino Superior de Uberaba- CESUBE

${ }^{3}$ Enfermeiro. Doutor em Ciências Sociais. Professor Associado do curso de Graduação em Enfermagem e dos Programas de Pós Graduação em Atenção à Saúde e Psicologia da UFTM.

${ }^{4}$ Mestre em Atenção à Saúde pela Universidade Federal do Triângulo Mineiro (UFTM). Doutorando em Atenção à Saúde.
} 
range $\geq 80$ years (mean mortality coefficient: $930.6 \times 10^{4}$ inhabitants) were more expressive. The general mortality coefficient $(\mathrm{p}=0.008)$ and by sex (male and female $-\mathrm{p}<0.001)$ showed an increasing temporal evolution. Conclusion: The importance of recognizing the mortality pattern due to diseases of the circulatory system as a public health problem and the creation of actions to prevent these diseases through the adoption of healthy lifestyles is highlighted.

Descriptors: Mortality; Cardiovascular System; Epidemiologic Studies; Time Series Studies.

\section{RESUMEN}

Objetivo: analizar la mortalidad por enfermedades del aparato circulatorio en un municipio del interior minero. Método: Se trata de estudio ecológico y serie temporal por coeficiente de mortalidad y análisis de tendencia. Se incluyeron los registros de muertes del capítulo IX Enfermedades del aparato circulatorio (CID-10: I00-I99) entre 1996 a 2014, disponibles en el Sistema de Información de Mortalidad, siendo emprendía análisis exploratorio descriptivo de las variables, además de análisis de tendencia temporal. Resultados: los fallecimientos para el sexo masculino (Coeficiente de mortalidad promedio: 82,2 $\times 10^{4}$ habitantes) y grupo de edad $\geq 80$ años (Coeficiente de mortalidad promedio: 930,6 × $10^{4}$ habitantes) fueron más expresivos. El coeficiente de mortalidad general $(\mathrm{p}=0,008)$ y por sexo (masculino y femenino - $\mathrm{p}<0,001)$ presentaron evolución temporal creciente. Conclusión: Se evidencia la importancia del reconocimiento del patrón de mortalidad por enfermedades del aparato circulatorio como problema de salud pública y la creación de acciones de prevención de estos agravios con adopción de estilos de vida saludables.

Descriptores: Mortalidad; Sistema Cardiovascular; Estudios Epidemiológicos; Estudios de Series Temporales.

\section{INTRODUÇÃO}

As condições de saúde da população são influenciadas por diversos fatores, que se relacionam de forma complexa. Observa-se, a nível global, uma melhora nas condições de vida e saúde das pessoas, com redução das taxas de mortalidade e aumento da longevidade. ${ }^{1}$ Neste contexto, emerge a transição epidemiológica, caracterizada pela mudança no padrão de morbimortalidade das doenças transmissíveis para as doenças crônicas não transmissíveis, em especial as Doenças do Aparelho Circulatório
(DAC). ${ }^{1,2}$
As DAC são as principais causas de óbitos em todo o mundo. Segundo a Organização Mundial de Saúde (OMS), as doenças do aparelho circulatório representaram cerca de 15,2 milhões de óbitos em todo o mundo, sendo as doenças isquêmicas do coração e acidente vascular encefálico as causas mais representativas. ${ }^{3}$

No Brasil, as DAC também tiveram grande representatividade frente a totalidade dos óbitos, correspondendo a cerca de $28 \%$ de todas as causas de óbito no ano de 2011. ${ }^{4}$ Estudo que avaliou a tendência de mortalidade na população adulta de um município mineiro, entre 1996 a 2013, identificou as DAC como 
principais causas dos óbitos, com média de idade do óbito de 59,7 anos. ${ }^{5}$

Embora as DAC representem uma das primeiras causas de morte no Brasil, a análise de tendência destes óbitos aponta redução desde a década de 1970. Apesar da redução, estes óbitos representam ainda grande impacto para a saúde pública do país, com custos elevados de internações e expressivas quantidades de óbitos. ${ }^{1,4-5}$

Neste contexto, o emprego de indicadores epidemiológicos de saúde, como análise da mortalidade por grupos de causas específicos e tendência destes óbitos, permitem análises das condições de saúde capazes de predizer o risco de adoecimento e situações de vulnerabilidade em saúde, que podem possuir como desfecho a morte. Ademais, a realização de estudos que visem conhecer o perfil de morbimortalidade é importante para a criação de diagnósticos em saúde que permitam a criação e readequação de ações que visem a prevenção destes agravos. Recente revisão aponta lacunas do conhecimento frente a realização de estudos que abordem a utilização de indicadores epidemiológicos na avaliação da mortalidade. ${ }^{2}$

Sendo assim, o presente estudo objetiva analisar a mortalidade por doenças do aparelho circulatório em um município do interior mineiro no período de 1996 a 2014.

\section{MÉTODO}

Trata-se de estudo ecológico que analisou a mortalidade por DAC da população adulta, residente no município de Uberaba/MG, no período de 1996 a 2014.

Este município é polo da Macrorregião de Saúde do Triângulo Sul, com população estimada de 330.361 habitantes para o ano de 2018. Estimativas do Departamento de Informática do Sistema Único de Saúde (DATASUS) apontam que a proporção da população adulta (igual ou maior que 20 anos) deste munícipio para o ano de 2012 foi de $72,29 \%$, o que denota a necessidade de estudos relacionados a esta população. ${ }^{6}$

Os dados foram obtidos por meio de informações registradas em banco de dados de domínio público do Sistema de Informação de Mortalidade (SIM), ${ }^{7}$ disponível no DATASUS.

A extração dos dados ocorreu entre os meses de setembro a outubro de 2018. O limite temporal proposto considera a atualidade dos dados disponíveis no DATASUS, conforme a Classificação Internacional de Doenças em sua $10^{\mathrm{a}}$ Revisão (CID-10). ${ }^{8}$ Foram utilizados as projeções intercensitárias disponibilizadas pelo DATASUS, sendo a populaçãopadrão considerada para o cálculo dos indicadores a do ano de $2010 .^{6}$ Os dados 
ignorados não compuseram a análise no presente estudo.

Foi organizado um banco de dados no Excel®, e realizada uma análise estatística exploratória descritiva das variáveis por medidas de tendência central e dispersão, além de uma análise dos indicadores e tendência temporal no software Statistical Package for the Social Sciences ${ }^{\circledR}$ (SPSS) versão 21.0.

As variáveis do estudo compreenderam os agrupamentos do capítulo IX da CID-10, conforme sexo e faixa etária, sendo o agrupamento utilizado a saber: I00-I02 Febre reumática aguda; I05-I09 Doenças reumáticas crônicas do coração; I10-I15 Doenças hipertensivas; I20-I25 Doenças isquêmicas do coração; I26-I28 Doenças cardíaca pulmonar e da circulação pulmonar; I30-I52 Outras formas de doença do coração; I60-I69 Doenças cerebrovasculares; I70-I79 Doenças das artérias, das arteríolas e dos capilares; I80-I89 Doenças das veias, dos vasos linfáticos e dos gânglios linfáticos, não classificadas em outra parte; I95-I99 Outros transtornos, e os não especificados do aparelho circulatório.

As informações foram analisadas em termos do Coeficiente de Mortalidade (CM) utilizando a expressão: Total de óbitos por DCV de residentes em certa área, no ano considerado/Populaçãopadrão residente na área * $10.000 .{ }^{9} \mathrm{Em}$ sequência, as informações foram analisadas para o CM específico $\left(10^{4}\right)$ : sexo (masculino e feminino), faixa etária, conforme disponibilizada pelo DATASUS (20 |-| 29 anos; 30 |- 39 anos; 40 |-| 49 anos; 50 |-| 59 anos; 60 |-| 69 anos; $70|-| 79$ anos e 80 anos ou mais). Os CM específicos foram confrontados em seu estado bruto ao longo do período. As taxas foram padronizadas segundo a população padrão deste estudo. Os resultados foram apresentados conforme valores anuais, trienais médios e intervalo de confiança de 95\% (IC95\%) para CM (total e específicos) no período.

A análise de tendência utilizou o modelo de regressão linear $(\mathrm{Y}=\beta 0+\beta 1 \mathrm{x}$ $+\sum$ ) no qual os valores da série do $\mathrm{CM}$ (total e específicos), foi considerado como variável dependente (y), e os períodos de estudo (anos) como variáveis independentes (x). Justifica-se esta opção de modelagem pela facilidade de elaboração, interpretação e poder estatístico. Para evitar a autocorrelação entre os termos da equação de regressão, foi utilizado o artifício de centralizar a variável ano, transformando-a em ano calendário menos ponto médio da série histórica. A tendência apresentou como medida de precisão o coeficiente de determinação $\left(\mathrm{R}^{2}\right)$ e a correlação de Pearson (r). A análise da série temporal poderia assumir tendência crescente, 
decrescente ou estacionária, admitindo-se tendência linear estatisticamente significativa com valor de $p \leq 0,05$. Foi identificada a presença de tendência nas séries como crescente ou decrescente conforme sinal determinante de $\beta 1$ (positivo ou negativo) e sentido da reta.

Conforme previsto pela resolução 510/2016 do Conselho Nacional de Pesquisa, como os dados utilizados são de domínio público não há necessidade de avaliação do estudo por Comitê de Ética em Pesquisa envolvendo seres humanos.

\section{RESULTADOS}

No período entre 1996 a 2014 ocorreram 10.111 óbitos para a faixa etária igual ou maior a 20 anos, em Uberaba, para as DAC. Sexo masculino (CM médio: 82,2 × $10^{4}$ hab.; IC95\%: 69,3; 95,0) e faixa etária $\geq 80$ anos (CM médio: 930,6 $2 \times 10^{4}$ hab.; IC95\%: 760,3; 1101,0) apresentaram as maiores médias do $\mathrm{CM}$ das variáveis analisadas (Tabela 1).

Tabela 1. Distribuição dos Coeficientes de Mortalidade $\left(10^{4}\right.$ habitantes $)$ por doenças do aparelho circulatório (CID-10: I00 a I99) conforme aspectos demográficos, 1996 a 2014, Uberaba-MG, Brasil, 2018.

\begin{tabular}{|c|c|c|c|c|c|c|c|c|}
\hline \multirow[b]{2}{*}{$\begin{array}{c}\text { Aspectos } \\
\text { demográficos }\end{array}$} & \multicolumn{6}{|c|}{ CM bruto no período entre 1996 a 2014} & \multirow[b]{2}{*}{ Média } & \multirow[b]{2}{*}{ IC 95\% } \\
\hline & $\begin{array}{l}1996- \\
1998\end{array}$ & $\begin{array}{l}1999- \\
2001\end{array}$ & $\begin{array}{l}2002- \\
2004\end{array}$ & $\begin{array}{l}2005- \\
2007\end{array}$ & $\begin{array}{c}2008- \\
2010\end{array}$ & $\begin{array}{l}2011- \\
2014\end{array}$ & & \\
\hline CM Geral & 69,6 & 73,7 & 67,9 & 73,5 & 79,0 & 103,5 & 77,9 & 67,$4 ; 88,4$ \\
\hline \multicolumn{9}{|l|}{ Sexo } \\
\hline Feminino & 66,6 & 69,9 & 65,5 & 70,3 & 76,5 & 93,7 & 73,7 & 65,$4 ; 82,1$ \\
\hline Masculino & 72,5 & 77,4 & 70,5 & 77,0 & 81,7 & 113,9 & 82,2 & 69,$3 ; 95,0$ \\
\hline \multicolumn{9}{|l|}{$\begin{array}{l}\text { Faixa etária } \\
\text { (anos) }\end{array}$} \\
\hline $20|-| 29$ & 2,3 & 1,9 & 1,2 & 2,1 & 3,4 & 1,8 & 2,1 & 1,$6 ; 2,7$ \\
\hline $30-139$ & 11,2 & 10,0 & 7,9 & 7,5 & 8,1 & 8,9 & 8,9 & 7,$8 ; 10,1$ \\
\hline $40|-| 49$ & 29,5 & 30,2 & 27,6 & 26,0 & 25,0 & 32,6 & 28,5 & 26,$2 ; 30,8$ \\
\hline $50 \mid-159$ & 62,0 & 63,8 & 54,6 & 64,1 & 66,5 & 81,8 & 65,5 & 58,$3 ; 72,7$ \\
\hline $60|-| 69$ & 165,4 & 175,6 & 145,6 & 187,3 & 172,1 & 240,3 & 181,1 & 155,$4 ; 206,8$ \\
\hline $70 \mid-19$ & 352,3 & 388,0 & 358,5 & 390,7 & 411,3 & 559,7 & 410,1 & 348,$9 ; 471,3$ \\
\hline$\geq 80$ & 757,5 & 812,2 & 857,1 & 808,3 & 1026,9 & 1321,7 & 930,6 & 760,$3 ; 1101,0$ \\
\hline
\end{tabular}

Fonte: SIM (DATASUS).

Com relação aos grupos de causas, conforme CID-10, evidenciam-se os grupos: $\quad$ I60 - I69 doenças cerebrovasculares (CM médio: 23,0 × $10^{4}$ hab.; IC95\%: 20,5; 25,5), I20-I25 doenças isquêmicas do coração (CM médio: 22,5 x
$10^{4}$ hab.; IC95\%: 16,5; 28,5), I30-I52 outras formas de doença do coração (CM médio: 19,0 x 104 hab.; IC95\%: 17,6; 19,3) e I10-I15 doenças hipertensivas (CM médio: 7,0 x $10^{4}$ hab.; IC95\%: 5,5; 8,5) 
com maiores valores médios no período do

estudo (Tabela 2).

Tabela 2. Distribuição dos Coeficientes de Mortalidade ( $10^{4}$ habitantes) conforme CID-10 (CID-10: I00 a I99), 1996 a 2014, Uberaba-MG, Brasil, 2018.

\begin{tabular}{|c|c|c|c|c|c|c|c|c|}
\hline \multirow{2}{*}{$\begin{array}{c}\text { Grupos } \\
\text { de } \\
\text { causas }\end{array}$} & \multicolumn{6}{|c|}{ CM bruto no período entre 1996 a 2014} & \multirow[b]{2}{*}{ Média } & \multirow[b]{2}{*}{ IC 95\% } \\
\hline & $\begin{array}{l}1996- \\
1998 \\
\end{array}$ & $\begin{array}{l}1999- \\
2001\end{array}$ & $\begin{array}{l}2002- \\
2004\end{array}$ & $\begin{array}{l}2005- \\
2007\end{array}$ & $\begin{array}{c}\text { 2008- } \\
2010\end{array}$ & $\begin{array}{l}2011- \\
2014\end{array}$ & & \\
\hline I00-I02 & - & 0,1 & - & - & 0,1 & 0,1 & 0,0 & 0,$0 ; 0,1$ \\
\hline I05-I09 & 1,5 & 0,4 & 0,4 & 0,9 & 0,6 & 1,0 & 0,8 & 0,$4 ; 1,1$ \\
\hline I10-I15 & 4,9 & 5,5 & 5,6 & 9,1 & 8,7 & 8,2 & 7,0 & 5,$5 ; 8,5$ \\
\hline I20-I25 & 20,4 & 18,8 & 16,8 & 19,2 & 22,4 & 37,3 & 22,5 & 16,$5 ; 28,5$ \\
\hline I26-I28 & 1,8 & 1,4 & 0,8 & 1,4 & 1,9 & 3,1 & 1,7 & 1,$1 ; 2,3$ \\
\hline I30-I52 & 18,5 & 20,1 & 17,1 & 17,9 & 18,1 & 19,0 & 19,0 & 17,$6 ; 19,3$ \\
\hline I60-I69 & 19,4 & 24,1 & 21,4 & 21,4 & 23,2 & 28,5 & 23,0 & 20,$5 ; 25,5$ \\
\hline I70-I79 & 2,6 & 2,6 & 4,9 & 3,1 & 3,3 & 4,4 & 3,5 & 2,$7 ; 4,3$ \\
\hline I80-I89 & 0,4 & 0,6 & 0,8 & 0,6 & 0,8 & 1,8 & 0,8 & 0,$4 ; 1,3$ \\
\hline I95-I99 & - & 0,1 & - & - & - & - & 0,0 & 0,$0 ; 0,1$ \\
\hline
\end{tabular}

Fonte: SIM (DATASUS).

Quanto à evolução temporal dos

óbitos por doenças do aparelho circulatório no período analisado, a regressão linear polinomial de primeira ordem apresentou coeficiente de determinação $\geq 70 \%$ para os CM de ambos os sexos e faixa etária 70 |-
79 anos e 80 anos ou mais. A tendência do CM geral dos óbitos analisados apresentou tendência crescente para o CM geral $(\mathrm{r}=0,5)$, representando uma correlação moderada (Tabela 3).

Tabela 3. Modelo de regressão da análise de tendência do CM x $10^{4}$ geral e das variáveis sexo e faixa etária, dos óbitos por doenças do aparelho circulatório, conforme CID-10 (CID10: I00 a I99), 1996 a 2014, Uberaba-MG, Brasil, 2018.

\begin{tabular}{llllll}
\hline Variáveis & Modelo & $\boldsymbol{p}$ & $\mathbf{R}^{2}$ & Tendência & $\mathbf{r}$ \\
\hline Geral & $\mathrm{y}=0,1889 \mathrm{x}+24,598$ & $\mathbf{0 , 0 0 8}$ & 0,3 & Crescente & 0,5 \\
Sexo Feminino & $\mathrm{y}=0,1295 \mathrm{x}+23,288$ & $\mathbf{< 0 , 0 0 1}$ & 0,8 & Crescente & 0,8 \\
Sexo Masculino & $\mathrm{y}=0,2599 \mathrm{x}+25,948$ & $<\mathbf{0 , 0 0 1}$ & 0,7 & Crescente & 0,9 \\
Faixa etária 20 |- 29 & $\mathrm{y}=-0,005 \mathrm{x}+0,6703$ & 0,06 & 0,2 & Decrescente & 0,4 \\
Faixa etária 30 |- 39 & $\mathrm{y}=-0,0809 \mathrm{x}+2,819$ & 0,19 & 0,4 & Decrescente & 0,2 \\
Faixa etária 40 |- 49 & $\mathrm{y}=-0,1119 \mathrm{x}+9,0032$ & 0,13 & 0,2 & Decrescente & 0,3 \\
Faixa etária 50 |- 59 & $\mathrm{y}=0,02 \mathrm{x}+20,682$ & $\mathbf{0 , 0 1}$ & 0,3 & Crescente & 0,5 \\
Faixa etária 60 |-| 69 & $\mathrm{y}=0,301 \mathrm{x}+57,176$ & $\mathbf{0 , 0 1}$ & 0,4 & Crescente & 0,6 \\
Faixa etária 70 |- 79 & $\mathrm{y}=1,3615 \mathrm{x}+129,5$ & $\mathbf{< 0 , 0 0 1}$ & 0,7 & Crescente & 0,8 \\
$\geq 80$ anos & $\mathrm{y}=5,4598 \mathrm{x}+293,88$ & $\mathbf{0 , 0 0 2}$ & 0,7 & Crescente & 0,6 \\
\hline
\end{tabular}

Fonte: SIM (DATASUS). 


\section{DISCUSSÃO}

O CM geral das DAC em Uberaba no período analisado apresentou tendência de crescimento, com destaque para o sexo masculino apresentando maior média do CM para todo o período analisado. As taxas de mortalidade para o sexo masculino no Brasil, entre 1990 e 2015, foi mais expressiva $(524,8$ e 315,8 , respectivamente), do que para o sexo feminino $\quad(358,3 \quad$ e 210,7 , respectivamente). ${ }^{10}$

Estudo que avaliou modelos de previsão a longo prazo de paciente portadores de doença cardiovascular com risco de morte identificou maiores chances de óbito com o incremento da idade. ${ }^{11}$ No presente estudo evidenciou-se maiores $\mathrm{CM}$ médio relacionados ao incremento de idade (faixas etárias). Resultados semelhantes também foram encontrados em estudo que comparou taxas de mortalidade por DAC no município de São Caetano do Sul, no estado de São Paulo e no Brasil, entre 1980 a 2010, em que maiores taxas foram encontradas para faixa etária mais longevas, com destaque para sexo masculino com idade superior a 60 anos. ${ }^{12}$

Tal situação pode estar relacionada a fatores como melhora na qualidade dos sistemas de informação em saúde no Brasil, com destaque para o preenchimento da declaração de óbito, declínio das causas mal definidas e aumento da prevalência de hipertensão arterial, em decorrência do melhor acesso na identificação do problema e controle pelos serviços de saúde. ${ }^{13}$

Os grupos de causas mais representativos (doenças cerebrovasculares, doenças isquêmicas do coração e doenças hipertensivas) encontrados nos resultados deste estudo corroboram com os achados de estudo ${ }^{13}$ que avaliou a evolução temporal das taxas de mortalidade por DAC no Brasil entre 1980 e 2012.

Com relação a evolução temporal, a tendência dos óbitos das doenças do aparelho circulatório apresentou tendência de crescimento, o que diverge dos resultados encontrados na literatura no que se refere a tendência deste grupo de causa, nos últimos anos para o Brasil ${ }^{10,14,15}$, estado do Acre $^{16}$ e municípios do estado do Rio de Janeiro ${ }^{4,17}$.

O reconhecimento dos padrões de morbi-mortalidade da população são fundamentais para análise do comportamento e tendência de determinados agravos, assim como para a análise das situações de saúde pelos gestores públicos, no que se refere a avaliação, planejamento e gestão das ações de promoção e prevenção pelos serviços de saúde, além de permitir redirecionamentos das políticas públicas em saúde., ${ }^{2,3}$

A morbimortalidade por doenças crônicas não transmissíveis, em especial as DAC, caracteriza-se com importante indicador de saúde no que se refere a efetividade dos serviços em saúde no Brasil, em especial aos serviços de Atenção Primária (AP). ${ }^{18,19}$ A utilização da estratégia de apoio matricial aos serviços de atenção primária tem sido destacada como inovadora e potencial no enfrentamento da problemática das doenças crônicas no país. Recente experiência de implementação de apoio matricial em cardiologia aos serviços da AP de município da região sul do país, evidenciou maior resolubilidade dos médicos da AP, com consequente redução e qualificação dos encaminhamentos para os cardiologistas, acesso mais rápido aos 
especialistas e aos exames, além de importante redução da fila de espera por atendimentos dessa especialidade. $^{20}$

Considerando as limitações deste estudo, por se tratar de estudo cuja fonte de dados se trata de dados secundários de domínio público proveniente do SIM, destaca-se a acurácia das informações. Outro aspecto se refere a metodologia empreendida relacionada ao delineamento ecológico, que não permite a análise de riscos individuais. Ademais, destaca-se a não redistribuição dos óbitos por causas mal definidas para o grupo das DAC e a generalização dos resultados por se tratar de um recorte local, que mesmo se tratando de realidade específica, pode ser importante fonte de informações como diagnóstico da situação de saúde da população.

\section{CONCLUSÃO}

Os resultados deste estudo corroboram para a importância do reconhecimento do padrão de mortalidade por DAC como problema de saúde pública. Apresenta perfil de mortalidade expressivo para o sexo masculino, faixa etária mais longevas e evolução temporal com distribuição linear crescente do CM x $10^{4}$ para as variáveis sexo e faixa etária de 50 anos e mais.

Para os serviços em saúde, mais do que mensurar o perfil dos óbitos por DAC, é necessário estimular a criação de ações de prevenção destes agravos e promoção à saúde com adoção de estilos de vida saudáveis. Neste sentido, estudos com delineamentos epidemiológicos emergem como estratégia de baixo custo e rápida execução na identificação deste problema que afeta a saúde dos indivíduos acometidos, e com isso contribuir na adoção de estratégias efetivas para a redução das mortes evitáveis por DAC.

\section{REFERÊNCIAS}

1. Moonesinghe R, Bouye K, PenmanAguilar A. Difference in health inequity between two population groups due to a social determinant in health. Int J Environ Res Public Health. [Internet]. 2014 [citado em 18 nov 2018]; 11(12):13074-83. doi: 10.3390/ijerph111213074

2. Garcia LAA, Camargo FC, Gomes THM, Rezende MP, Araújo GA, Iwamoto $\mathrm{HH}$, et al. Produção do conhecimento de enfermagem sobre os anos potenciais de vida perdidos: estudo bibliométrico.

REFACS [Internet]. 2017 [citado em 23 out 2018]; 5(1):34-46. doi: http://dx.doi.org/10.18554/refacs.v5i1.191 1

3. World Health Organization. The top 10 causes of death [Internet]. Geneva: WHO; 2018 [citado em 23 out 2018]. Disponível em: http://www.who.int/news-room/factsheets/detail/the-top-10-causes-of-death 4. Soares GP, Klein CH, Souza e Silva NA, Oliveira GMM. Evolution of cardiovascular diseases mortality in the counties of the state of Rio de Janeiro from 1979 to 2010. Arq Bras Cardiol.

[Internet]. 2015 [citado em 23 out 2018]; 104(5):356-65. doi: http://dx.doi.org/10.5935/abc.20150019 5. Garcia LA, Camargo F, Pereira G, Ferreira LA, Iwamoto H, Santos Álvaro, et al. Anos potenciais de vida perdidos e tendência de mortalidade na população adulta em um município do Triângulo Mineiro, 1996-2013. Medicina (Ribeirão Preto). [Internet]. 2017 [citado 23out.2018]; 50(4):216-25. Disponível em: http://www.revistas.usp.br/rmrp/article/vie w/140485/135463

6. Ministério da Saúde (Brasil).

DATASUS, Informações de Saúde, Informações demográficas e socioeconômicas. Brasília, DF: Ministério 
da Saúde; 2019. Disponível em:

http://www.datasus.gov.br.

7. Ministério da Saúde (Brasil).

DATASUS, Informações de Saúde,

Estatísticas Vitais. Brasília, DF: Ministério da Saúde; 2019. Disponível em:

http://www.datasus.gov.br.

8. Organização Mundial de Saúde, Centro

Brasileiro de Classificação de Doenças em

Português. Classificação estatística

internacional de doenças e problemas

relacionados à saúde. 10ed rev. São Paulo:

EDUSP; 1998.

9. Medronho RA. Estudos ecológicos. In:

Medronho RA, Bloch KV, Luiz RR,

Werneck GL, editores. Epidemiologia. São

Paulo: Atheneu; 2009. p. 265-74.

10. Brant LCC, Nascimento BR, Passos

VMA, Duncan BB, Bensenõr IJM, Malta

DC, et al. Variações e diferenciais da

mortalidade por doença cardiovascular no

Brasil e em seus estados, em 1990 e 2015:

estimativas do Estudo Carga Global de

Doença. Rev Bras Epidemiol. [Internet].

2017 [citado em 19 nov 2018]; 20(Supl

1):116-28. Disponível em:

https://www.scielosp.org/pdf/rbepid/2017. v20suppl1/116-128/pt

11. Hata J, Nagai A, Hirata M, Kamatani

Y, Tamakoshi A, Yamagata Z, et al. Risk prediction models for mortality in patients with cardiovascular disease: the BioBank Japan Project. J Epidemiol. [Internet]. 2017 [citado em 19 nov 2018];

27(3S):S71-S76. Disponível em: https://www.ncbi.nlm.nih.gov/pmc/articles /PMC5350588/

12. Luz FE, Santos BRM, Sabino W.

Estudo comparativo de mortalidade por doenças cardiovasculares em São Caetano do Sul (SP), Brasil, no período de 1980 a 2010. Ciênc Saúde Colet. [Internet]. 2017 [citado em 19 nov 2018]; 22(1):161-68.

Disponível em:

http://dx.doi.org/10.1590/1413-

81232017221.18362015

13. Morais RM, Costa AL. Uma avaliação do Sistema de Informações sobre

Mortalidade. Saúde Debate [Internet].

2017 [citado 23out 2018]; 41(esp):101-17.
Disponível em:

https://www.scielosp.org/article/ssm/conte nt/raw/?resource_ssm_path=/media/assets/ sdeb/v41nspe/0103-1104-sdeb-41-nspe0101.pdf

14. Villela PB, Klein CH, Oliveira GMM.

Evolução da mortalidade por doenças cerebrovasculares e hipertensivas no Brasil entre 1980 e 2012. Arq Bras Cardiol. [Internet]. 2016[citado em 19 nov 2018]; 107(1):26-32. Disponível em:

http://www.scielo.br/pdf/abc/v107n1/pt_00 66-782X-abc-20160092.pdf

15. Guimarães RM, Andrade SSCA, Machado EL, Bahia CA, Oliveira MM, Jacques FVL. Diferenças regionais na transição da mortalidade por doenças cardiovasculares no Brasil, 1980 a 2012. Rev Panam Salud Publica [Internet]. 2015 [citado em 19 nov 2018]; 37(2):83-9.

Disponível em:

https://www.scielosp.org/pdf/rpsp/2015.v3 7n2/83-89/pt

16. Bezerra PCL, Monteiro GTR.

Tendência de mortalidade geral e por doenças do aparelho circulatório em idosos, Rio Branco, Acre, 1980-2012. Rev Bras Geriatr Gerontol. [Internet]. 2018 [citado em 19 nov 2018]; 21(2):145-57. Disponível em: http://www.scielo.br/pdf/rbgg/v21n2/pt_18 09-9823-rbgg-21-02-00143.pdf

17. Soares GP, Klein CH, Souza e Silva NA, Oliveira GMM. Evolução da mortalidade por doenças do aparelho circulatório e do produto interno bruto per capita nos municípios do estado do Rio de Janeiro. Int J Cardiovasc Sci. [Internet]. 2018 [citado em 19 nov 2018]; 31(2):12332. Disponível em:

http://publicacoes.cardiol.br/portal/ijcs/port ugues/2018/v3102/pdf/3102006.pdf

18. Souza DK, Peixoto SV. Estudo descritivo da evolução dos gastos com internações hospitalares por condições sensíveis à atenção primária no Brasil, 2000-2013. Epidemiol Serv Saúde [Internet]. 2017 [citado em 19 nov 2018]; 26(2):285-94. doi: 
http://dx.doi.org/10.5123/s1679-

49742017000200006

19. GBD 2013 Mortality and Causes of

Death Collaborators. Global, regional, and

national age-sex specific all-cause and

cause-specific mortality for 240 causes of

death, 1990-2013: a systematic analysis for

the Global Burden of Disease Study 2013.

Lancet [Internet]. 2015 [citado em 19 nov

2018]; 385(9963):117-71. Disponível em:

https://www.ncbi.nlm.nih.gov/pmc/articles

/PMC4340604/

20. Hoepfner C, Franco SC, Maciel RA,

Hoepfner AMS. Programa de apoio

matricial em cardiologia: qualificação e

diálogo com profissionais da atenção

primária. Saúde Soc. [Internet]. 2014

[citado em 19 nov 2018]; 23(3):1091-101.

Disponível em:

http://www.scielo.br/pdf/sausoc/v23n3/010

4-1290-sausoc-23-3-1091.pdf

RECEBIDO: 22/11/18

APROVADO: $21 / 03 / 19$

PUBLICADO: 07/2019 